\title{
SCIENTIFIC REPORTS

\section{OPEN The Association between Body Composition using Dual energy X-ray Absorptiometry and Type-2 \\ Diabetes: A Systematic Review and Meta-Analysis of Observational studies}

Received: 22 May 2019

Accepted: 20 August 2019

Published online: 02 September 2019

\author{
Preeti Gupta ${ }^{1}$, Carla Lanca ${ }^{1}{ }^{1}$, Alfred T. L. Gan ${ }^{1}$, Pauline Soh ${ }^{1}$, Sahil Thakur ${ }^{1}$, Yijin Tao ${ }^{1}$, \\ Neelam Kumari ${ }^{1}$, Ryan E. K. Man ${ }^{1,2}$, Eva K. Fenwick ${ }^{1,2}$ \& Ecosse L. Lamoureux ${ }^{1,2}$
}

The association between objective measures of body composition (BC) with type 2 diabetes (T2DM) is inconclusive. We conducted a systematic review and meta-analysis to examine the association between several body composition (BC) indices assessed using dual energy X-ray absorptiometry (DXA), and T2DM. Using PRISMA guidelines, we searched for observational studies investigating BC measures, including total body fat mass (BFM), visceral fat mass (VFM), subcutaneous fat mass (SFM), and fat free mass (FFM); and T2DM. Of 670 titles initially identified, 20 were included. High VFM was consistently associated with T2DM. For every kg increase in VFM, the odds of having T2DM increased by two-fold for males (OR 2.28 [95\% CI 1.42 to 3.65], $\mathrm{p}=0.001$ ) and more than 4-fold for females (OR 4.24 [1.64 to 11.02], $p=0.003$ ). The presence of T2DM was associated with 2 -fold higher odds of low FFM (OR 2.38 [1.44 to 3.95]). We found evidence that greater VFM is a risk factor for prevalent and incident T2DM. While the presence of T2DM is associated with reduced FFM; the relationship between FFM and BFM with T2DM remains unclear. Reducing VFM and increasing FFM through lifestyle changes may reduce the risk of T2DM and mitigate its deleterious effect on $B C$, respectively.

The prevalence of diabetes mellitus (DM) is rapidly increasing worldwide, with 592 million people expected to have the condition by $2035^{1}$. As such, the prevention and management of DM has become a crucial public health concern with an emphasis on addressing modifiable risk factors, such as poor diet, sedentary lifestyle, and obesity $^{2}$

While the relationship between obesity and DM, using surrogate anthropometric measures such as body mass index $[\mathrm{BMI}]^{3,4}$, waist circumference $[\mathrm{WC}]^{5,6}$, and waist-hip ratio $[\mathrm{WHR}]^{7,8}$, have been widely studied, results have been equivocal ${ }^{9,10}$, as these proxy measures are unable to distinguish body composition (BC) indices such as fat tissue deposition from muscle mass and bone density. Furthermore, these surrogate measures do not provide information on the location of fat mass deposition, emphasizing the need to evaluate the contribution of objective components of $\mathrm{BC}$ on DM risk.

Specific BC measurements can be estimated indirectly using various objective techniques such as air displacement plethysmography (BodPod), bioelectrical impedance analysis (BIA) ${ }^{11}$, and dual energy X-ray absorptiometry (DXA). However, BC profiles measured by different objective techniques are not interchangeable, as studies have reported poor concordance between these different methods ${ }^{12-14}$. In this analysis we only included studies that assessed $\mathrm{BC}$ measures using DXA, which is commonly used ${ }^{15}$, and is an acceptable technique for measuring $\mathrm{BC}$ in clinical studies ${ }^{16,17}$ with a substantially lower cost, no complex post processing requirements, and minimal

${ }^{1}$ Singapore Eye Research Institute, Singapore and Singapore National Eye Centre, Singapore, Singapore. ${ }^{2}$ Duke-NUS Medical School, Singapore, Singapore. Correspondence and requests for materials should be addressed to E.L.L. (email: ecosse.lamoureux@seri.com.sg) 
radiation exposure $(\sim 1 \mu \mathrm{SV})$ compared to computed tomography (CT) or magnetic resonance imaging (MRI) $\operatorname{scan}^{18}$. There is increasing evidence that DXA-assessed BC measures are associated with the onset, progression, treatment response, and health outcomes of cardiometabolic diseases, including type 2 DM (T2DM) ${ }^{19-22}$.

To date, there is no comprehensive review on the relationship between DXA-assessed BC measures, including total body fat mass (BFM), visceral fat mass (VFM), subcutaneous fat mass (SFM), muscle mass (MM), and the presence and incidence of DM. A better understanding of this association is critical as it can inform clinical guidelines and interventions for the management of DM. We therefore performed a systematic review and meta-analysis of observational studies evaluating the bidirectional associations between objectively assessed BC measures (i.e. BFM including VFM and SFM; and MM) and the prevalence or incidence of T2DM. We also identified key knowledge gaps and suggest future research directions.

\section{Methods}

Literature search. We performed a systemic review and comprehensive literature search using three sources (PubMed, Web of Science and the Cochrane Central Register of Controlled Trials) for English language research articles published between January 1998 and August 2018. The databases were systematically searched using a combination of the following keywords and Boolean operators: body composition OR body fat mass OR visceral fat mass OR subcutaneous fat mass OR adiposity OR visceral adipose tissue OR fat free mass OR muscle mass OR lean body mass OR skeletal muscle mass AND type 2 diabetes. The studies included observational epidemiological studies in T2DM populations such as cohort, case control and cross-sectional studies, in which DXA was utilized to assess BC in humans. This process continued until a search saturation point was found; i.e. the point at which additional terms showed no improvement in our search result. Relevant references identified from the bibliographies of pertinent articles were also retrieved.

Study selection. Using our search strategy, 670 titles were initially identified. Two authors (PG and CL) assessed the titles independently according to predefined inclusion criteria. Studies were then systematically excluded after detailed examination, if the title and abstract were not relevant. Any potential disagreements were resolved through consulting the senior author (EL). If necessary, full-text articles of studies were also obtained, particularly if there was insufficient information within the abstract to determine exclusion.

Inclusion criteria. Eligibility criteria were based on the PICOS (participants, intervention, comparability, outcomes, study design) framework recommended by the PRISMA guidelines ${ }^{23}$.

1. Study Type: We included observational studies (cross-sectional, case-control and prospective).

2. Participants: Studies that included human participants with T2DM.

3. Exposures: Four objective BC measurements (total BFM, VFM, SFM, and MM) obtained using DXA were selected as the exposures of interest. For MM, we included studies which reported total lean mass (TLM) or appendicular skeletal muscle mass (ASM) - two of the most commonly used measures of MM. Furthermore, we accepted studies reporting on BFM or MM and DM as either exposure/outcome.

4. Outcomes: Outcomes were the prevalence or incidence of T2DM. We accepted studies using different T2DM assessment methods, including but not limited to: random glucose $\geq 11.1 \mathrm{mmol} / \mathrm{L}, \mathrm{HbA} 1 \mathrm{c} \geq 6.5 \%$ $(\geq 48 \mathrm{mmol} / \mathrm{moL})$, fasting plasma glucose $>125 \mathrm{mg} / \mathrm{dl}$ or $\geq 7.0 \mathrm{mmol} / \mathrm{l}$, self-reported use of oral hypoglycaemic medications or insulin, and/or history of physician-diagnosed diabetes.

Exclusion criteria. The following types of papers were excluded:

1. Conference abstracts.

2. Papers not written in the English language.

3. Studies on animals, and in-vitro/in-vivo studies.

4. Studies involving individuals with type $1 \mathrm{DM}$.

5. Studies in people without diabetes, or those with participants with impaired glucose tolerance or pre-diabetes.

6. Studies measuring biomarkers of BC in serum, blood, or urine without any link to BC assessment using DXA.

7. Exposures which were assessed using objective methods other than DXA, such as bioelectrical impedance analysis (BIA), MRI or CT.

8. Studies only measuring outcomes of "cardiometabolic diseases" or "metabolic risk factors" without specific reference to T2DM.

9. Articles with insufficient data to draw conclusions. This included any form of data insufficiency which did not enable us to draw conclusions from/evaluate the study, (e.g. lack of exposure/outcome definitions, or lack of statistical analysis).

10. For our meta-analysis, we also excluded studies that did not report the required statistical parameters and which we could not obtain despite repeated attempts to contact the study authors.

Data extraction. A standardized data extraction form based on the "Strengthening the Reporting of Observational Studies in Epidemiology" (STROBE) statement ${ }^{24}$ was used to extract the following relevant data from each included article: authors, year, study design, sample size, population characteristics, age of participants, objective BC measures, DM outcome type, method of T2DM diagnosis, adjustment for confounders used in analysis, statistical methods used, and summary of key findings. Data extraction was done by one author (PG) and confirmed by another (CL). 


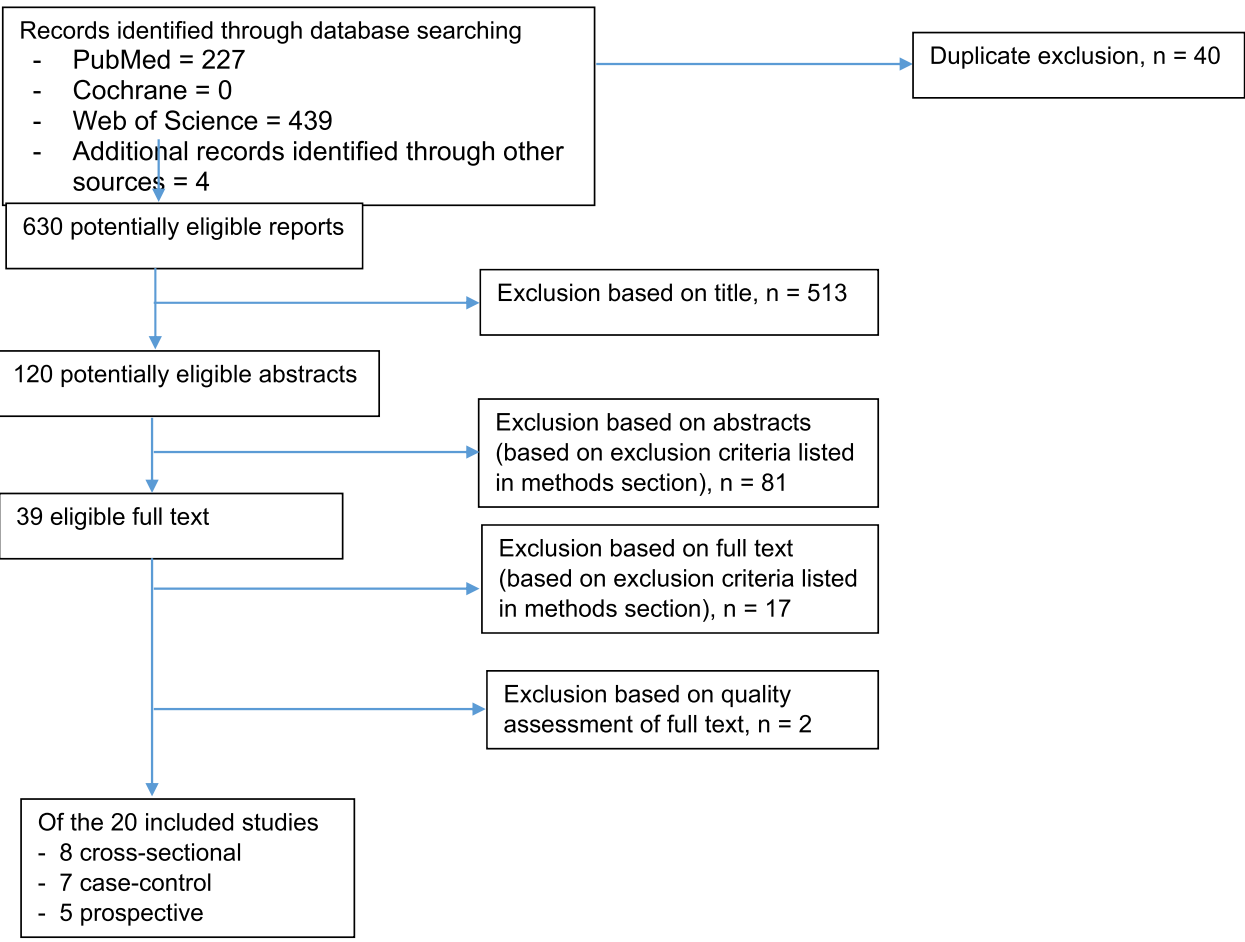

Figure 1. PRISMA Flow Diagram: Selection of included studies.

Study quality evaluation. The quality of observational studies was assessed using a modified version of the Newcastle Ottawa Scale (NOS), a validated tool for evaluating observational study designs ${ }^{25}$. Originally designed to assess prospective and case-control studies, an adapted version of the NOS was used in the current study to allow the assessment of cross-sectional studies ${ }^{26}$. The NOS uses three main bias-reducing criteria to award up to a maximum of 9 stars: (a) the selection and representativeness of the participants (maximum of 4 stars), (b) the comparability of groups (maximum of 2 stars), and (c) the ascertainment of exposure (for case-control) or outcome (for prospective and cross-sectional) (maximum of 3 stars). Given that BMI or WC are important confounders in the association between BC and T2DM, we gave studies a star (under comparability criteria) if they adjusted for BMI or WC in their analyses. Studies assigned $0-4,5-6$, and $\geq 7$ stars were considered as low, medium and high quality (low risk of bias) respectively. Studies with low quality (i.e. high risk of bias) were excluded from this review.

Statistical analysis. All statistical analyses were performed using Intercooled Stata version 15 for Windows (StataCorp, Lake Station, TX). Due to the paucity of studies reporting on the total BFM-T2DM relationship, and no studies on SFM, we could not perform meta-analysis of these BC measures and T2DM. We conducted random effects meta-analysis to pool the crude odds ratios (ORs) relating VFM and MM with T2DM, including studies that provided this information. ORs were calculated from two-way contingency tables where they were not reported but cell counts were available. We chose not to pool adjusted ORs as studies varied substantially in their adjustment for potential confounders which might have affected the direction and significance of the associations reported. Given that BC varies by gender, the meta-analysis of VFM-T2DM was stratified by gender. In contrast, no stratification was done for the meta-analysis of MM-T2DM as too few studies provided gender-stratified data. Heterogeneity was quantified using the I-squared statistic. A higher I-squared value meant greater heterogeneity in study effects. We reported all pooled estimates with $95 \%$ confidence intervals and judged a p-value $<0.05$ as statistically significant.

\section{Results}

Description of studies. Of 670 titles screened, 120 were extracted for detailed evaluation, of which 20 adhered to our inclusion criteria (Fig. 1). They comprised five prospective, eight cross-sectional, and seven case-control studies. The majority had high NOS scores, with 19 classified as "high quality" ( $\geq 7$ stars) and one as "moderate quality" (5 stars). No study was excluded because of "low quality" (Tables 1 and 2).

Association of BFM and its components with T2DM. Total BFM as the exposure. The association between total BFM and T2DM was equivocal ${ }^{27-30}$. In a cross-sectional study of Korean adults aged 50 years or older, Choi and colleagues reported increased total body fat \% in both men $(23.44 \pm 4.91$ vs $21.98 \pm 5.23$, $\mathrm{p}<0.001)$ and postmenopausal women $(34.85 \pm 5.05$ vs $33.85 \pm 5.59, \mathrm{p}<0.001)$ with T2DM compared to those without $^{29}$. In contrast, in a longitudinal study, Neeland and associates found no association between total BFM or body fat $\%$ and incident $\mathrm{T}_{2} \mathrm{DM}^{30}$. However, as both studies used simple univariate methods, the lack of multivariate adjustment for potential confounders, may bias their results. 


\begin{tabular}{|c|c|c|c|c|c|c|c|c|}
\hline Author, year & Association & Study design & Quality & Study population & Sample size & $\begin{array}{l}\text { DM (outcome) } \\
\text { assessment method }\end{array}$ & $\begin{array}{l}\text { Analysis and } \\
\text { variables } \\
\text { adjusted for }\end{array}$ & Main findings \\
\hline Choi, 2017 & $\begin{array}{l}\text { Total body fat } \\
\% \text { was higher } \\
\text { in individuals } \\
\text { with T2DM }\end{array}$ & Cross-sectional & 8 & Asians (Korean) & $\begin{array}{l}6575(3027 \\
\text { males, } 3548 \\
\text { postmenopausal } \\
\text { females })\end{array}$ & $\begin{array}{l}\text { Fasting glucose } \\
\geq 126 \mathrm{mg} / \mathrm{dL} \\
(7.0 \mathrm{mmol} / \mathrm{L}), \\
\text { medical diagnosis, } \\
\text { use of oral } \\
\text { hypoglycaemic } \\
\text { medications or } \\
\text { insulin injections }\end{array}$ & T-test & $\begin{array}{l}\text { Total body fat } \% \\
\text { was higher in both } \\
\text { men }(23.44 \pm 4.91 \\
\text { vs } 21.98 \pm 5.23 \text {, } \\
\text { p }<0.001) \text { and women } \\
(34.85 \pm 5.05 \text { vs } \\
33.85 \pm 5.59, p<0.001) \\
\text { with T2DM than those } \\
\text { without. }\end{array}$ \\
\hline Neeland, 2012 & $\begin{array}{l}\text { No difference } \\
\text { in total fat } \\
\text { mass (kg) } \\
\text { and body } \\
\text { fat } \%\end{array}$ & Prospective & 8 & $\begin{array}{l}\text { Caucasians (United } \\
\text { States) }\end{array}$ & $\begin{array}{l}732(256 \text { men, } 476 \\
\text { women) }\end{array}$ & $\begin{array}{l}\text { Prevalent medical } \\
\text { treatment for } \\
\text { T2DM, fasting } \\
\text { blood glucose } \\
\geq 126 \mathrm{mg} / \mathrm{dL} \\
(7.0 \mathrm{mmol} / \mathrm{L}) \text {,or } \\
\text { non-fasting blood } \\
\text { glucose } \geq 200 \mathrm{mg} / \mathrm{dl}\end{array}$ & $\begin{array}{l}\text { Chi square and } \\
\text { Wilcoxon rank- } \\
\text { sum test }\end{array}$ & $\begin{array}{l}\text { No difference in total } \\
\text { fat mass ( } 35.3 \text { vs } 35.5, \\
\mathrm{p}=0.51) \text { and body } \\
\text { fat } \%(39.8 \text { vs } 40.4, \\
\mathrm{p}=0.51) \text { among those } \\
\text { with incident T2DM } \\
\text { compared to no DM }\end{array}$ \\
\hline Raska, 2016 & $\begin{array}{l}\text { No difference } \\
\text { in fat mass/ } \\
\text { height }^{2} \text { and } \\
\text { fat mass \% }\end{array}$ & Case-control & 5 & Caucasians (Australians) & $\begin{array}{l}139 \\
\text { postmenopausal } \\
\text { women ( } 68 \text { with } \\
\text { T2DM, } 71 \text { age-and } \\
\text { weight-matched } \\
\text { without T2DM) }\end{array}$ & $\begin{array}{l}\text { Biochemical } \\
\text { analyses of HbAlc } \\
\text { and serum fasting } \\
\text { glucose }\end{array}$ & $\begin{array}{l}\text { T-test } \\
\text { Age-and weight- } \\
\text { matched }\end{array}$ & $\begin{array}{l}\text { No difference in fat } \\
\text { mass/height }{ }^{2}(14.4 \pm 5.5 \\
\text { vs } 13.35 \pm 5.2) \text { and fat } \\
\text { mass } \%(41.12 \pm 4.9 \\
\text { vs } 41.19 \pm 5.97) \text { in } \\
\text { postmenopausal } \\
\text { women with and } \\
\text { without T2DM }\end{array}$ \\
\hline Stoney, 1998 & $\begin{array}{l}\text { No difference } \\
\text { in total fat } \\
\text { mass }\end{array}$ & Case-control & 8 & Caucasians & $\begin{array}{l}84 \text { postmenopausal } \\
\text { women ( } 42 \text { T2DM, } \\
42 \text { non-DM, age } \\
\text { and BMI matched) }\end{array}$ & $\begin{array}{l}\text { Current DM } \\
\text { medication or } \\
\text { abnormal OGTT }\end{array}$ & $\begin{array}{l}\text { T-test } \\
\text { Age and BMI }\end{array}$ & $\begin{array}{l}\text { No difference in total } \\
\text { fat mass }(30.9 \pm 1.5 \\
\text { vs } 31.8 \pm 1.5) \text { in } \\
\text { postmenopausal } \\
\text { women with and } \\
\text { without T2DM } \\
\end{array}$ \\
\hline Heshka, 2008 & $\begin{array}{l}\text { T2DM was } \\
\text { associated } \\
\text { with reduced } \\
\text { total BFM }\end{array}$ & Cross-sectional & 7 & $\begin{array}{l}\text { Caucasians (Black, White } \\
\text { and Hispanics) }\end{array}$ & $\begin{array}{l}1315 \text { ( } 490 \text { men, } 825 \\
\text { women) }\end{array}$ & & $\begin{array}{l}\text { Multivariable } \\
\text { Regression } \\
\text { Age, gender, } \\
\text { race, clinical site, } \\
\text { height, weight } \\
\text { and body size } \\
\end{array}$ & $\begin{array}{l}\text { Less total fat mass } \\
(-1.4 \pm 0.3[\text { S.E.]; } 34.5 \\
\text { vs } 35.8 \mathrm{~kg}, \mathrm{p}<0.001) \text { in } \\
\text { T2DM than controls }\end{array}$ \\
\hline Nordstrom, 2016 & $\begin{array}{l}\text { Higher VFM } \\
\text { increased } \\
\text { the risk of } \\
\text { T2DM }\end{array}$ & Cross-sectional & 7 & Caucasians (Swedish) & $\begin{array}{l}1393 \text { ( } 705 \text { men, } 688 \\
\text { women) }\end{array}$ & $\begin{array}{l}\text { Fasting-plasma } \\
\text { glucose level of } \\
\text { at least } 7 \mathrm{mmol} / \mathrm{L} \\
\text { or based on } \\
\text { questionnaire } \\
\text { responses }\end{array}$ & \begin{tabular}{|l|} 
Logistic \\
Regression \\
Smoking, PA, \\
SBP, DBP, \\
cholesterol, \\
HDL, TG, \\
previous MI and \\
stroke
\end{tabular} & $\begin{array}{l}\text { Per SD increase in } \\
\text { VFM/body weight was } \\
\text { the strongest predictor } \\
\text { of T2DM in men } \\
(\mathrm{OR}=3.64,95 \% \mathrm{CI} \text { : } \\
2.53-5.25) .\end{array}$ \\
\hline Rothney, 2013 & $\begin{array}{l}\text { Higher VFM } \\
\text { increased } \\
\text { the risk of } \\
\text { T2DM }\end{array}$ & Cross-sectional & 7 & Caucasians (Italian) & $\begin{array}{l}939 \text { ( } 541 \text { men, } 398 \\
\text { women) }\end{array}$ & $\begin{array}{l}\text { Fasting plasma } \\
\text { glucose }>125 \mathrm{mg} / \mathrm{dl}\end{array}$ & $\begin{array}{l}\text { Multivariable } \\
\text { Linear } \\
\text { Regression } \\
\text { Age, BMI and } \\
\text { WC }\end{array}$ & $\begin{array}{l}\text { The OR (per SD } \\
\text { change in VFM) for } \\
\text { T2DM }=2.07 \text { ( } 95 \% \mathrm{CI} \text { : } \\
0.73-5.87 \text { ) for women } \\
\text { and } 2.25 \text { (95\% CI: } \\
1.21-4.19 \text { ) for men. }\end{array}$ \\
\hline Jung, 2016 & $\begin{array}{l}\text { Higher VFM } \\
\text { increased } \\
\text { the risk of } \\
\text { T2DM }\end{array}$ & Cross-sectional & 7 & Asians (Korean) & $\begin{array}{l}1603 \text { ( } 611 \text { men, } 992 \\
\text { women) }\end{array}$ & $\begin{array}{l}\text { Fasting glucose } \\
\geq 126 \mathrm{mg} / \mathrm{dL} \\
(7.0 \mathrm{mmol} / \mathrm{L}), 2-\mathrm{h} \\
\text { glucose } \geq 200 \mathrm{mg} / \\
\text { dL }(11.1 \mathrm{mmol} / \mathrm{L}) \\
\text { during the OGTT } \\
(75 \mathrm{~g}), \mathrm{HbA} 1 \mathrm{c} \text { level } \\
\geq 6.5 \%, \text { or use of } \\
\text { hypoglycaemic } \\
\text { medications }\end{array}$ & $\begin{array}{l}\text { Multivariable } \\
\text { Logistic } \\
\text { Regression } \\
\text { Age, alcohol } \\
\text { consumption, } \\
\text { PA, education } \\
\text { and menopause } \\
\text { (for females) }\end{array}$ & $\begin{array}{l}\text { VFM in the upper } 10^{\text {th }} \\
\text { percentile had highest } \\
\text { OR for DM (men: } \\
\text { OR=15.9, } 95 \% \text { CI: } 6.4- \\
39.2 \text {; women: OR }=6.9 \text {, } \\
\text { 95\%CI: } 3.5-13.7) \text {. } \\
\text { VFM had the highest } \\
\text { AUC with DM (men: } \\
0.69,95 \% \text { CI: } 0.64-0.73 \text {; } \\
\text { women: } 0.70,95 \% \text { CI: } \\
0.67-0.74) .\end{array}$ \\
\hline
\end{tabular}

Table 1. Summary of data extracted from the eight observational studies on the association between total, and visceral fat mass and diabetes included in the systematic review. T2DM: type-2 diabetes, DM: diabetes mellitus; BFM: body fat mass; VFM: visceral fat mass; FFM: fat free mass; ASM: appendicular skeletal mass; BMI: body mass index; WC: waist circumference; PA: physical activity; SBP: systolic blood pressure; DBP: diastolic blood pressure; TG: triglyceride; HDL: high density cholesterol; LDL: low density cholesterol; HRT: hormone replacement therapy; MI: myocardial infarction; OR: odds ratio; CI: confidence interval; HR: hazard ratio; SD: standard deviation; AUC: area under curve.

Total BFM as the outcome. We found only one cross-sectional study of 1315 Caucasians (mean age 58.5 \pm 6.6 ), published study by Heska and colleagues, who reported that subjects with T2DM had reduced total BFM, compared to those without $(34.5 \mathrm{vs} 35.8 \mathrm{~kg}, \mathrm{p}<0.001)^{20}$. 


\begin{tabular}{|c|c|c|c|c|c|c|c|c|}
\hline Author, year & Association & Study Design & Quality & Study Population & $\begin{array}{l}\text { Sample } \\
\text { Size }\end{array}$ & $\begin{array}{l}\text { DM outcome } \\
\text { assessment }\end{array}$ & $\begin{array}{l}\text { Analysis and } \\
\text { variables } \\
\text { adjusted for }\end{array}$ & Main Findings \\
\hline Kim, 2014 & $\begin{array}{l}\text { Low muscle } \\
\text { mass measures } \\
\text { in men with } \\
\text { T2DM }\end{array}$ & Case-control & 7 & Asians (Koreans) & $\begin{array}{l}414(189 \\
\text { men, } 225 \\
\text { women); } \\
144 \text { T2DM, } \\
270 \\
\text { controls }\end{array}$ & $\begin{array}{l}\text { Onset of DM after } \\
\text { age } 25 \text { years, use of } \\
\text { oral hypoglycaemic } \\
\text { medications or insulin, } \\
\text { or fasting plasma glucose } \\
\geq 126 \mathrm{mg} / \mathrm{dL} \text {. }\end{array}$ & $\begin{array}{l}\text { Multivariable } \\
\text { Logistic } \\
\text { Regression } \\
\text { Age, BMI, } \\
\text { current smoking, } \\
\text { SBP, DBP, total } \\
\text { cholesterol, TG, } \\
\text { HDL }\end{array}$ & $\begin{array}{l}\text { Older men with T2DM } \\
\text { had 2-4 times increased } \\
\text { risk of low muscle mass } \\
\text { measures (ASM/height } \\
\text { ASM/weight and total } \\
\text { skeletal muscle/weight; } \\
\text { OR range }=2.63-4.45 \text { ). }\end{array}$ \\
\hline Kim, 2010 (KSOS) & $\begin{array}{l}\text { Low ASM/ } \\
\text { height }^{2} \text { in } \\
\text { T2DM }\end{array}$ & Case-control, & 8 & Asians (Koreans) & $\begin{array}{l}810(414 \\
\text { DM, 396 } \\
\text { controls) }\end{array}$ & Not defined & $\begin{array}{l}\text { Multivariable } \\
\text { Logistic } \\
\text { Regression } \\
\text { Age, gender, } \\
\text { BMI, smoking, } \\
\text { alcohol, PA, } \\
\text { SBP, DBP, } \\
\text { medications and } \\
\text { lipid profile }\end{array}$ & $\begin{array}{l}\text { T2DM had higher risk } \\
\text { of low ASM/height }{ }^{2} \\
\text { (OR }=3.06,95 \% \text { CI: } \\
1.42-6.62) \text { than those } \\
\text { without. }\end{array}$ \\
\hline Anbalagan, 2013 & $\begin{array}{l}\text { Low ASM/ } \\
\text { height }^{2} \text { in } \\
\text { T2DM }\end{array}$ & Case-control & 8 & Asians (Indians) & $\begin{array}{l}152(72 \\
\text { T2DM and } \\
72 \text { age-sex } \\
\text { matched } \\
\text { controls })\end{array}$ & $\begin{array}{l}\text { Fasting plasma glucose } \\
\text { and } 2 \text {-h post load }(75 \mathrm{~g}) \\
\text { plasma glucose }\end{array}$ & $\begin{array}{l}\text { Multivariable } \\
\text { Logistic } \\
\text { Regression } \\
\text { Age, gender, diet, } \\
\text { PA, smoking, } \\
\text { alcohol, DM }\end{array}$ & $\begin{array}{l}\text { T2DM was associated } \\
\text { with increased risk of low } \\
\text { ASM/height }(\mathrm{OR}=6.01, \\
\text { 95\% CI: } 1.34-26.88), \\
\text { compared to those } \\
\text { without. } \\
\text { The relationship became } \\
\text { insignificant on further } \\
\text { adjustment for HbA1c } \\
\text { or fasting plasma } \\
\text { glucose } \mathrm{OR}=3.29, \\
95 \% \mathrm{CI}: 0.629-17.28 \\
\text { and OR }=3.94,95 \% \mathrm{CI} \\
0.794-19.65 \text {, respectively). }\end{array}$ \\
\hline Guerroro, 2016 & $\begin{array}{l}\text { Lower ASM/ } \\
\text { BMI in women } \\
\text { with T2DM }\end{array}$ & Case-control & 7 & $\begin{array}{l}\text { Caucasians (United } \\
\text { states) }\end{array}$ & $\begin{array}{l}139 \text { adults } \\
\text { (88 women, } \\
51 \text { men); } \\
100 \text { T2DM } \\
\text { and 39 } \\
\text { controls }\end{array}$ & $\begin{array}{l}\text { Selected T2DM patients } \\
\text { with over } 4 \text { years on oral } \\
\text { anti diabetic drugs or } \\
\text { insulin }\end{array}$ & $\begin{array}{l}\text { T-test or Mann } \\
\text { Whitney }\end{array}$ & $\begin{array}{l}\text { Women with T2DM had } \\
\text { significantly lower ASM/ } \\
\text { BMI (5.3 [4.4-8]) than } \\
\text { those without }(5.9[4.2-8] \text {; } \\
\mathrm{p}=0.02) \text {. }\end{array}$ \\
\hline Moon, 2014 & $\begin{array}{l}\text { Low ASM/ } \\
\text { weight in non- } \\
\text { obese T2DM } \\
\text { adults }\end{array}$ & Cross-sectional & 7 & Asians (Koreans) & $\begin{array}{l}10432 \\
\text { adults } \\
(4558 \\
\text { men, } 5874 \\
\text { women })\end{array}$ & $\begin{array}{l}\text { Previously diagnosed } \\
\text { T2DM, use of anti- } \\
\text { hyperglycaemic } \\
\text { medication, or fasting } \\
\text { plasma } \geq 100 \mathrm{mg} / \mathrm{dL}\end{array}$ & $\begin{array}{l}\text { Multivariable } \\
\text { Logistic } \\
\text { Regression } \\
\text { Age, sex, region, } \\
\text { smoking, alcohol } \\
\text { consumption, } \\
\text { exercise, family } \\
\text { income and BMI }\end{array}$ & $\begin{array}{l}\text { In older }(\geq 60 \mathrm{yrs}) \\
\text { non-obese, those with } \\
\text { low ASM/weight had } \\
\text { higher risk of T2DM } \\
(\mathrm{OR}=2.44,95 \% \mathrm{CI} \\
1.69-3.53, \mathrm{p}<0.001) . \\
\text { This was not significant } \\
\text { in obese }(\mathrm{OR}=1.26,95 \% \\
\text { CI } 0.76-2.10, \mathrm{p}=0.362) \\
\text { individuals. }\end{array}$ \\
\hline Yoon, 2016 & $\begin{array}{l}\text { No association } \\
\text { between ASM/ } \\
\text { height }^{2} \text { and } \\
\text { T2DM }\end{array}$ & Cross-sectional & 7 & Asians (Koreans) & $\begin{array}{l}269 \text { men } \\
(79 \text { T2DM, } \\
190 \\
\text { controls })\end{array}$ & $\begin{array}{l}\text { HbAlc } \geq 6.5 \% \text { or } \\
\text { current use of insulin } \\
\text { or oral hypoglycaemic } \\
\text { medication }\end{array}$ & $\begin{array}{l}\text { T-test } \\
\text { Age, smoking, } \\
\text { alcohol, PA, } \\
\text { BMI, duration } \\
\text { of DM }\end{array}$ & $\begin{array}{l}\text { No significant difference } \\
\text { in ASM/height }{ }^{2} \text { between } \\
\text { subjects with or without } \\
\text { T2DM }(7.46 \pm 0.77 \text { vs } \\
7.39 \pm 0.85, \mathrm{p}=0.563)\end{array}$ \\
\hline Akeroyd, 2014 & $\begin{array}{l}\text { Lower ASM in } \\
\text { T2DM }\end{array}$ & Cross-sectional & 8 & $\begin{array}{l}\text { Caucasians (United } \\
\text { states) }\end{array}$ & $\begin{array}{l}1137 \\
\text { men }(142 \\
\text { T2DM, } 995 \\
\text { controls })\end{array}$ & $\begin{array}{l}\text { Self-report of physician } \\
\text { diagnosed or use of oral } \\
\text { hypoglycaemic agents }\end{array}$ & $\begin{array}{l}\text { Multivariable } \\
\text { Linear } \\
\text { Regression } \\
\text { Age, race, BMI, } \\
\text { PA }\end{array}$ & \begin{tabular}{|l|} 
Men with T2DM had \\
significantly lower \\
ASM (mean deviation \\
{$[\mathrm{MD}]=-1.04 \mathrm{~kg}$,} \\
$\mathrm{p}=0.04)$ than those \\
without. \\
No significant difference \\
in leg lean mass.
\end{tabular} \\
\hline Davidson, 2014 & $\begin{array}{l}\text { Lower FFM in } \\
\text { T2DM }\end{array}$ & Cross-sectional & 7 & $\begin{array}{l}\text { Caucasians (United } \\
\text { states) }\end{array}$ & $\begin{array}{l}171(95 \\
\text { T2DM, } 76 \\
\text { controls) }\end{array}$ & Physician diagnosis & \begin{tabular}{|l} 
General linear \\
models \\
Height, weight, \\
age, sex and race
\end{tabular} & $\begin{array}{l}\text { Adjusted FFM was } \\
\text { significantly lower in } \\
\text { those with T2DM than } \\
\text { controls }(\mathrm{p}<0.05)\end{array}$ \\
\hline $\begin{array}{l}\text { Larsen, 2016: The } \\
\text { Health ABC study }\end{array}$ & $\begin{array}{l}\text { Greater FFM } \\
\text { is associated } \\
\text { with lower } \\
\text { incidence of } \\
\text { DM for older } \\
\text { normal-weight } \\
\text { women but } \\
\text { not for men } \\
\text { or overweight } \\
\text { women. }\end{array}$ & Prospective & 8 & $\begin{array}{l}\text { Caucasians (United } \\
\text { states) }\end{array}$ & $\begin{array}{l}2076(202 \\
\text { incident } \\
\text { T2DM); } \\
958 \text { men, } \\
20176 \\
\text { women }\end{array}$ & $\begin{array}{l}\text { Physician diagnosed, use } \\
\text { of oral hypoglycaemic } \\
\text { agents or insulin with } \\
\text { onset after age } 25 \text { years, } \\
\text { fasting plasma glucose } \\
\geq 7.0 \mathrm{mmol} / \mathrm{L}\end{array}$ & $\begin{array}{l}\text { Cox Regression } \\
\text { Age, race, } \\
\text { clinical site, } \\
\text { PA, smoking, } \\
\text { lipid profile, } \\
\text { hypertension } \\
\text { and VFM }\end{array}$ & $\begin{array}{l}\text { High FFM was not } \\
\text { associated with lower } \\
\text { risk of incident T2DM } \\
\text { (HR }=0.37,95 \% \text { CI: } \\
0.17-0.83 \text { ) in normal } \\
\text { weight women. } \\
\text { Higher levels of the FFM } \\
\text { was associated with } \\
\text { greater risk of incident } \\
\text { T2DM for overweight/ } \\
\text { obese (total FFM: } \\
\text { HR }=1.10,95 \% \text { CI: } \\
0.89-1.36 \text { ) women. } \\
\text { No associations seen in } \\
\text { men }\end{array}$ \\
\hline
\end{tabular}




\begin{tabular}{|c|c|c|c|c|c|c|c|c|}
\hline Author, year & Association & Study Design & Quality & Study Population & $\begin{array}{l}\text { Sample } \\
\text { Size }\end{array}$ & $\begin{array}{l}\text { DM outcome } \\
\text { assessment }\end{array}$ & $\begin{array}{l}\text { Analysis and } \\
\text { variables } \\
\text { adjusted for }\end{array}$ & Main Findings \\
\hline $\begin{array}{l}\text { Park, 2009, The } \\
\text { Health ABC study }\end{array}$ & $\begin{array}{l}\text { T2DM is } \\
\text { associated } \\
\text { with excessive } \\
\text { loss of total } \\
\text { muscle mass } \\
\text { and ASM }\end{array}$ & Prospective & 7 & $\begin{array}{l}\text { Caucasians (United } \\
\text { states) }\end{array}$ & $\begin{array}{l}2675(1324 \\
\text { men, } 1351 \\
\text { women); } \\
628 \text { T2DM, } \\
20147 \\
\text { controls }\end{array}$ & $\begin{array}{l}\text { Physician diagnosed, use } \\
\text { of oral hypoglycaemic } \\
\text { agents or insulin with } \\
\text { onset after age } 25 \text { years, } \\
\text { fasting plasma glucose } \\
\geq 7.0 \mathrm{mmol} / \mathrm{L} \text {, or a } 2-\mathrm{h} \\
\text { post challenge plasma } \\
\text { glucose } \geq 11.1 \mathrm{mmol} / \mathrm{l}\end{array}$ & $\begin{array}{l}\text { Generalized } \\
\text { Estimating } \\
\text { Equation } \\
\text { Age, sex, race, } \\
\text { clinic site, } \\
\text { baseline BMI, } \\
\text { weight loss }\end{array}$ & $\begin{array}{l}\text { The rate of decline in total } \\
\text { muscle mass }(-186 \pm 25 \\
\text { vs }-125 \pm 7, \mathrm{p}<0.05) \\
\text { and ASM }(-149 \pm 14 \text { vs } \\
-113 \pm 4, \mathrm{p}<0.05) \text { was } \\
\text { greater in older adults } \\
\text { with undiagnosed T2DM, } \\
\text { than in those without. }\end{array}$ \\
\hline $\mathrm{Li}, 2016$ & $\begin{array}{l}\text { Reduced } \\
\text { FFM \& ASM/ } \\
\mathrm{ht}^{2} \text { is not a } \\
\text { risk factor } \\
\text { for incident } \\
\text { T2DM in men }\end{array}$ & Prospective & 8 & Caucasians (Australians) & $\begin{array}{l}1632 \\
\text { men }(146 \\
\text { incident } \\
\text { T2DM, } \\
1486 \\
\text { controls) }\end{array}$ & $\begin{array}{l}\text { Previous doctor } \\
\text { diagnosis, antiglycemic } \\
\text { medication use, } \\
\text { fasting plasma glucose } \\
\geq 7.0 \mathrm{mmol} / \mathrm{L}(\geq 126 \mathrm{mg} / \\
\mathrm{dl}), \text { or } \mathrm{HbAlc} \geq 6.5 \% \\
(48 \mathrm{mmol} / \mathrm{mol}) .\end{array}$ & $\begin{array}{l}\text { Multivariable } \\
\text { Logistic } \\
\text { Regression } \\
\text { Age, income, } \\
\text { cohort, WC, } \\
\text { fasting plasma } \\
\text { glucose, PA, } \\
\text { hypertension, } \\
\text { TG, family } \\
\text { history of DM, } \\
\text { and grip strength }\end{array}$ & $\begin{array}{l}\text { Reduced FFM \& ASM/ } \\
\mathrm{ht}^{2} \text { were not significant } \\
\text { risk factors for T2DM } \\
\text { incidence (per } 5 \mathrm{~kg} \text { unit } \\
\text { increase in FFM and per } \\
1 \mathrm{~kg} / \mathrm{m}^{2} \text { increase in ASM/ } \\
\mathrm{ht}^{2} \text { on T2DM incidence, } \\
\mathrm{OR}=1.03,95 \% \text { CI:0.87- } \\
1.24 \text {; OR }=1.08: 95 \% \mathrm{CI} \text { : } \\
0.83-1.39 \text {, respectively). }\end{array}$ \\
\hline Renoud, 2014 & $\begin{array}{l}\text { T2DM is } \\
\text { associated } \\
\text { with faster } \\
\text { muscle loss in } \\
\text { older men }\end{array}$ & Prospective & 7 & Caucasians (French) & 608 men & Glycemia $\geq 100 \mathrm{mg} / \mathrm{dL}$ & $\begin{array}{l}\text { Multivariable } \\
\text { Linear } \\
\text { Regression } \\
\text { Age, testosterone } \\
\text { and PA }\end{array}$ & $\begin{array}{l}\text { Men with T2DM had } \\
\text { higher age-related } \\
\text { acceleration of muscle } \\
\text { loss versus men without } \\
(-0.08 \text { vs }-0.03 \% \text { /year/ } \\
\text { age, } \mathrm{p}<0.05)\end{array}$ \\
\hline
\end{tabular}

Table 2. Summary of data extracted from the 12 observational studies on the association between fat free mass and diabetes included in the systematic review. T2DM: type-2 diabetes, DM: diabetes mellitus; VFM: visceral fat mass; FFM: fat free mass; ASM: appendicular skeletal mass; BMI: body mass index; WC: waist circumference; PA: physical activity; SBP: systolic blood pressure; DBP: diastolic blood pressure; TG: triglyceride; HDL: high density cholesterol; OR: odds ratio; CI: confidence interval.

VFM as the exposure. We found evidence of a strong association between accumulation of VFM and prevalent T2DM, after adjusting for relevant confounders ${ }^{31-35}$. In a cross-sectional sample of 939 Italian men and women, VFM was independently associated with a two-fold risk of having T2DM, even after adjustment for BMI and $\mathrm{WC}^{35}$. Similarly, Nordstrom and colleagues demonstrated almost a four-fold (OR=3.64, 95\% CI: 2.53-5.25) and 1.5 -fold $(\mathrm{OR}=1.41,95 \% \mathrm{CI}$ : 0.93-2.13) higher odds of having T2DM in men and women for every SD increase in VFM/body weight, respectively ${ }^{33}$.

Subcutaneous Fat Mass (SFM) as the exposure or outcome. We found no published data on the relationship between SFM (assessed using DXA) and T2DM.

Association of FFM with T2DM. MM as the outcome. Of twelve relevant studies, MM was the outcome in nine of them ${ }^{21,36-46}$. Of these, eight (five case control, one cross-sectional, and two longitudinal) found an independent association between T2DM and reduced $\mathrm{MM}^{36-43}$. For instance, in a case-control study, Kim and associates, reported that even after adjusting for relevant covariates older ( $>65$ years) Korean men with T2DM had a 2-4 fold increased odds of low muscle mass measures (ASM/height ${ }^{2}$, ASM/weight, and TSM/weight) ${ }^{40}$. Similarly, Anbalagan and colleagues found that Asian Indians with T2DM had significantly increased odds of low ASM $/ \mathrm{ht}^{2}(\mathrm{OR}=6.01,95 \% \mathrm{CI}$ : $1.34-26.88)$ than those without $^{37}$, after adjusting for age, gender, diet, physical activity, smoking, alcohol, DM duration and treatment, although the relationship was attenuated when HbAlc was introduced into the model $(\mathrm{OR}=3.94,95 \% \mathrm{CI}$ : 0.79-19.65).

In the Health, ABC Study of community-dwelling older adults (70-79 years), Parks and colleagues found that the rate of decline in TLM and ASM (gram/year) was most profound in older adults with undiagnosed T2DM (TLM: $-186 \pm 25$; ASM: $-149 \pm 14$ ), followed by diagnosed T2DM (TLM: $-106 \pm 20$; ASM: $-130 \pm 11$ ) than in those without DM (TLM: $-125 \pm 7$; ASM: $-113 \pm 4$ ), even after adjusting for changes in body weight over time ${ }^{42}$.

$M M$ as the exposure. The association between MM and T2DM was equivocal ${ }^{21,45,46}$. For instance, in the Korean National Health and Nutrition Examination Survey (KNHANES), low muscle mass was found to be an early marker for T2DM (individuals with low ASM/wt had higher odds of T2DM (OR =2.44, 95\% CI: 1.69-3.53; $\mathrm{p}<0.001)$ than those without ${ }^{21}$. Conversely, in a fully-adjusted prospective, population-based, randomly selected cohort of men (mean age $54.1 \pm 11.4$ years), Li and associates found that reduced TLM and ASM/ht ${ }^{2}$ were not significant risks factors for incident T2DM (per $5 \mathrm{~kg}$ increase in TLM and per $1 \mathrm{~kg} / \mathrm{m}^{2}$ increase in ASM/ $\mathrm{ht}^{2}$ on T2DM incidence, $\mathrm{OR}=1.03,95 \% \mathrm{CI}: 0.87-1.24$; OR $=1.08: 95 \%$ CI: 0.83-1.39, respectively $)^{46}$.

Meta-analysis of the association of VFM with T2DM. VFM as the exposure. For the VFM-T2DM relationship, three cross-sectional studies were included in the meta-analysis ${ }^{31,33-35}$. We found that for every $\mathrm{kg}$ increase in VFM, the odds of T2DM increased more than two-fold for males (OR $=2.28,95 \%$ CI: 1.42 to 3.65, $\mathrm{p}=0.001)$ and more than 4 -fold for females $(\mathrm{OR}=4.24,95 \% \mathrm{CI}: 1.64$ to $11.02, \mathrm{p}=0.003$; Fig. 2$)$. 


\begin{tabular}{|c|c|c|c|c|}
\hline \multirow{2}{*}{\multicolumn{2}{|c|}{ Study }} & \multirow[b]{2}{*}{ OR $(95 \% \mathrm{Cl})$} & \multicolumn{2}{|l|}{$\%$} \\
\hline & & & Weight & $\mathrm{N}$ \\
\hline \multicolumn{5}{|l|}{ Male } \\
\hline Rothney (2013) & $=$ & $1.60(1.35,1.90)$ & 36.02 & 398 \\
\hline Jung (2016) & $\rightarrow$ & $2.37(1.63,3.45)$ & 30.54 & 611 \\
\hline Nordstrom (2016) & $=$ & $3.23(2.44,4.27)$ & 33.44 & 705 \\
\hline Subtotal $(I-$ squared $=89.3 \%, p=0.000)$ & & $2.28(1.42,3.65)$ & 100.00 & \\
\hline \multicolumn{5}{|l|}{ Female } \\
\hline Rothney (2013) & $\rightarrow$ & $2.31(1.59,3.36)$ & 34.38 & 541 \\
\hline Jung (2016) & & $12.20(7.05,21.12)$ & 32.41 & 992 \\
\hline Nordstrom (2016) & - & $2.84(1.76,4.62)$ & 33.21 & 688 \\
\hline Subtotal $(I-$ squared $=92.1 \%, p=0.000)$ & & $4.24(1.64,11.02)$ & 100.00 & \\
\hline NOTE: Weights are from random effects & alysis & & & \\
\hline
\end{tabular}

Figure 2. Forest plot* of the crude odds ratio of diabetes per $1 \mathrm{~kg}$ increase in visceral fat mass. *The size of the box of each study effect corresponds to the relative weight given to that study in the meta-analysis; the diamond refers to the overall pooled estimates with $95 \%$ confidence interval.

\begin{tabular}{|c|c|c|c|}
\hline \multirow[b]{2}{*}{ Study } & \multicolumn{3}{|c|}{$\%$} \\
\hline & OR $(95 \% \mathrm{Cl})$ & Weight & $\mathrm{N}$ \\
\hline $\operatorname{Kim}(2010)$ & $=2.54(1.58,4.07)$ & 23.44 & 810 \\
\hline Anbalagan (2013) & $-3.48(1.61,7.50)$ & 17.52 & 152 \\
\hline Kim (2014) & $1.19(0.75,1.88)$ & 23.69 & 414 \\
\hline Moon (2014) & $3.58(2.86,4.49)$ & 27.82 & 10432 \\
\hline Guerrero (2016) & $1.61(0.33,7.93)$ & 7.54 & 139 \\
\hline Overall $(I-$ squared $=78.7 \%, p=0.001)$ & $2.38(1.44,3.95)$ & 100.00 & \\
\hline NOTE: Weights are from random effects analysis & & & \\
\hline $\begin{array}{ll}1 & \\
.2 & 1\end{array}$ & 8 & & \\
\hline
\end{tabular}

Figure 3. Forest plot* of the crude odds ratio of diabetes presence to low appendicular skeletal muscle mass. *The size of the box of each study effect corresponds to the relative weight given to that study in the metaanalysis; the diamond refers to the overall pooled estimates with $95 \%$ confidence interval.

Meta-analysis of the association of MM with T2DM. Estimates in the form of ORs were available from four studies with low MM as the outcome $\mathrm{e}^{37,39-41}$ and one study with T2DM as the outcome ${ }^{21}$. In line with our systematic review, our meta-analysis presented ORs of MM. We found presence of T2DM was associated with 2.4 times higher odds of low MM (ASM/ht²; crude OR 2.38 [1.44 to 3.95], p=0.001; Fig. 3).

\section{Discussion}

In this systematic review and meta-analysis of the relationship between DXA- measured BC indices and T2DM, we found a consistent evidence of an association between high levels of visceral fat and the risk of T2DM, as well as a significant association between T2DM presence and low MM, after accounting for traditional diabetes risk factors. These findings suggest that reducing VFM and improving MM through lifestyle changes such as diet and physical activity, may reduce the risk of T2DM, and mitigate the deleterious effect of the condition on MM. In contrast, the relationship between total BFM, MM and T2DM, remains unclear, while no studies to date have explored the relationship between DXA-assessed SFM and T2DM. Longitudinal studies are however needed to better understand the temporality and mechanisms underlying the relationships between total BFM, SFM and $\mathrm{MM}$; and T2DM. 
Our finding that VFM may increase the risk of $\mathrm{T} 2 \mathrm{DM}^{31,33,35}$ could be explained by several potential underlying mechanisms. First, increased VFM is related to low adiponectin levels ${ }^{47}$. Adiponectin plays a pivotal role in energy metabolism, and has antiglycemic (increases insulin sensitivity), anti-inflammatory, antiangiogenic, and cardio protective properties ${ }^{48,49}$. Therefore, reduced secretion of adiponectin in individuals with high fat mass, particularly VFM, may lead to a cascade of biochemical reactions including an increase in insulin resistance and impaired glucose homeostasis (by augmenting hepatic gluconeogenesis and inhibiting glucose uptake in skeletal muscles) ${ }^{50}$ resulting in hyperglycaemia. Second, lower adiponectin levels increases the secretion of pro-inflammatory cytokines (such as c-reactive proteins [CRP], interlukins and tumor necrosis factor [TNF] alpha $)^{51}$, which have been linked to the pathogenesis of T2DM. Third, the accumulation of visceral fat has been suggested to have lipolytic potential. This may, in turn, result in an increased delivery of free fatty acid to the liver's portal circulation, which may induce hepatic insulin resistance by stimulating gluconeogenesis and interfering with hepatic insulin removal ${ }^{50}$.

Given that the relationship between total BFM and T2DM remain inconsistent, future studies are needed. While there is some evidence in studies using CT or MRI to measure subcutaneous fat ${ }^{52}$, data on the relationship between DXA-assessed SFM and T2DM are lacking. This is likely because previous versions of DXA were unable to compartmentalise SFM. Further studies using newer DXA models which can provide SFM in a large, population-based sample across the spectrum of BMI are warranted to assess the impact of SFM on the prevalence and incidence of T2DM. Studies unequivocally showed that people with T2DM were more likely to have lower $\mathrm{MM}^{36-43}$ through several mechanisms, such as decreased glucose utilization by muscle ${ }^{53}$, increased levels of systemic inflammatory cytokines such as interlukin-6, TNF-alpha, and C-reactive protein ${ }^{54,55}$ oxidative stress $^{56}$, and mitochondrial dysfunction ${ }^{57}$. However, the relationship between reduced MM and risk of developing T2DM is still unclear ${ }^{21,45,46}$, and further studies are needed to determine this relationship and associated underpinning mechanisms.

There is substantial evidence indicating percent body fat differs between Asian and Western populations ${ }^{58,59}$, suggesting that the body composition profiles of patient with T2DM might also differ in these populations. As such, we also analysed various BC parameters separately in Asians and Caucasians. For BFM, most of the included studies ( 6 out of 8) were from Caucasians, limiting our ability to conclude whether objective fat measures differed between Asians and Caucasians. Of the 9 studies (4 Asians, 5 Caucasians) included in the systematic review of the DM-MM relationship, we found that T2DM was associated with low MM measures in both populations. However, there were only 3 studies (1 Asian, 2 Caucasians) for MM-DM relationship, with equivocal findings, suggesting the need for more studies to untangle this relationship in general, and between different populations.

There are several strengths of our systematic review. First, we only evaluated the relationship between BC measures and T2DM in human subjects, which allows for a more direct translation of results into clinical recommendations for patients with T2DM. Second, the studies in our review had wide geographic diversity, which can aid its generalizability. Third, we did not limit the timeframe, allowing a broad range of literature from 1998 to 2018. Finally, we excluded studies involving people with T1DM. This is important as there are pathophysiological, etiological, epidemiological and disease management differences between diabetes types which means that the influence of BC may differ between T1DM and T2DM.

While the majority of studies included in our review had high NOS scores, several limitations must be highlighted. First, eight studies were cross-sectional, which limit their ability to establish a causal relationship between $\mathrm{BC}$ and T2DM. The relatively low number of longitudinal studies $(\mathrm{n}=5)$ included in the analysis, out of the 20 available in the literature, demonstrates the need for more well-designed cohort studies. Second, we excluded non-English publications which may have resulted in some publication bias. Third, as data from only eight articles were included in the meta-analysis, the generalizability of these findings might be limited. Fourth, the studies in our systematic review varied in their adjustment for potential confounders, thus we chose not to pool the adjusted odds ratio. Our meta-analysis summarised crude odds ratios, therefore our observed associations may be confounded by characteristics strongly related to T2DM risk that vary depending on body composition, such as diabetes duration and physical activity. Last, most studies only assessed a single BC parameter, and did not consider the interrelation between the key BFM measures (including both VFM and SFM) and MM. Future studies should assess the different BC phenotypes like low fat-high muscle mass, high fat-high muscle mass, low fat-low muscle mass, and high fat-low muscle mass to better reflect real world BC outcomes, which can then be translated into clearer clinical BC guidelines ${ }^{60-64}$.

In conclusion, our systematic review and meta-analysis demonstrated that higher BFM, particularly VFM, is associated with greater T2DM risk, and conversely the presence of T2DM was associated with a likelihood of lower MM. However, the relationship between MM (exposure) and T2DM remains unclear. Our findings suggest that optimal diabetes management and reducing VFM and increasing MM through lifestyle changes in the form of a more balanced diet and increased physical activity, may reduce the risk of T2DM and mitigate its deleterious impact on BC, respectively. However, further prospective studies to untangle the relationship between total BFM, SFM, and MM, on T2DM are needed in order to better inform clinical guidelines for disease prevention and management.

\section{References}

1. Guariguata, L. et al. Global estimates of diabetes prevalence for 2013 and projections for 2035. Diabetes Res Clin Pract 103, 137-149, https://doi.org/10.1016/j.diabres.2013.11.002 (2014).

2. Feldman, A. L. et al. Change in lifestyle behaviors and diabetes risk: evidence from a population-based cohort study with 10 year follow-up. The international journal of behavioral nutrition and physical activity 14, 39, https://doi.org/10.1186/s12966-017-0489-8 (2017).

3. Nguyen, N. T., Nguyen, X. M., Lane, J. \& Wang, P. Relationship between obesity and diabetes in a US adult population: findings from the National Health and Nutrition Examination Survey, 1999-2006. Obes Surg 21, 351-355, https://doi.org/10.1007/s11695-0100335-4 (2011). 
4. Narayan, K. M., Boyle, J. P., Thompson, T. J., Gregg, E. W. \& Williamson, D. F. Effect of BMI on lifetime risk for diabetes in the U.S. Diabetes care 30, 1562-1566, https://doi.org/10.2337/dc06-2544 (2007).

5. Feller, S., Boeing, H. \& Pischon, T. Body mass index, waist circumference, and the risk of type 2 diabetes mellitus: implications for routine clinical practice. Dtsch Arztebl Int 107, 470-476, https://doi.org/10.3238/arztebl.2010.0470 (2010).

6. Siren, R., Eriksson, J. G. \& Vanhanen, H. Waist circumference a good indicator of future risk for type 2 diabetes and cardiovascular disease. BMC Public Health 12, 631, https://doi.org/10.1186/1471-2458-12-631 (2012).

7. Schmidt, M. I., Duncan, B. B., Canani, L. H., Karohl, C. \& Chambless, L. Association of waist-hip ratio with diabetes mellitus. Strength and possible modifiers. Diabetes care 15, 912-914 (1992).

8. Vazquez, G., Duval, S., Jacobs, D. R. Jr. \& Silventoinen, K. Comparison of body mass index, waist circumference, and waist/hip ratio in predicting incident diabetes: a meta-analysis. Epidemiol Rev 29, 115-128, https://doi.org/10.1093/epirev/mxm008 (2007).

9. Gomez-Ambrosi, J. et al. Body adiposity and type 2 diabetes: increased risk with a high body fat percentage even having a normal BMI. Obesity (Silver Spring. Md.) 19, 1439-1444, https://doi.org/10.1038/oby.2011.36 (2011).

10. Solanki, J. D., Makwana, A. H., Mehta, H. B., Gokhale, P. A. \& Shah, C. J. Body Composition in Type 2 Diabetes: Change in Quality and not Just Quantity that Matters. Int J Prev Med 6, 122, https://doi.org/10.4103/2008-7802.172376 (2015).

11. Lee, S. Y. \& Gallagher, D. Assessment methods in human body composition. Current opinion in clinical nutrition and metabolic care 11, 566-572, https://doi.org/10.1097/MCO.0b013e32830b5f23 (2008).

12. Pateyjohns, I. R., Brinkworth, G. D., Buckley, J. D., Noakes, M. \& Clifton, P. M. Comparison of three bioelectrical impedance methods with DXA in overweight and obese men. Obesity (Silver Spring, Md.) 14, 2064-2070, https://doi.org/10.1038/oby.2006.241 (2006).

13. Leahy, S., O'Neill, C., Sohun, R. \& Jakeman, P. A comparison of dual energy X-ray absorptiometry and bioelectrical impedance analysis to measure total and segmental body composition in healthy young adults. European journal of applied physiology 112, 589-595, https://doi.org/10.1007/s00421-011-2010-4 (2012).

14. Neovius, M., Hemmingsson, E., Freyschuss, B. \& Udden, J. Bioelectrical impedance underestimates total and truncal fatness in abdominally obese women. Obesity (Silver Spring, Md.) 14, 1731-1738, https://doi.org/10.1038/oby.2006.199 (2006).

15. Andreoli, A., Scalzo, G., Masala, S., Tarantino, U. \& Guglielmi, G. Body composition assessment by dual-energy X-ray absorptiometry (DXA). La Radiologia medica 114, 286-300, https://doi.org/10.1007/s11547-009-0369-7 (2009).

16. Going, S. B. et al. Detection of small changes in body composition by dual-energy x-ray absorptiometry. Am J Clin Nutr 57, 845-850, https://doi.org/10.1093/ajen/57.6.845 (1993).

17. Chen, Z. et al. Dual-energy X-ray absorptiometry is a valid tool for assessing skeletal muscle mass in older women. J Nutr 137, 2775-2780, https://doi.org/10.1093/jn/137.12.2775 (2007).

18. Sasai, H. et al. Does Visceral Fat Estimated by Dual-Energy X-ray Absorptiometry Independently Predict Cardiometabolic Risks in Adults? J Diabetes Sci Technol 9, 917-924, https://doi.org/10.1177/1932296815577424 (2015).

19. Bragg, F. et al. Associations of General and Central Adiposity With Incident Diabetes in Chinese Men and Women. Diabetes care 41, 494-502, https://doi.org/10.2337/dc17-1852 (2018).

20. Heshka, S. et al. Altered body composition in type 2 diabetes mellitus. International journal of obesity (2005) 32, 780-787, https:// doi.org/10.1038/sj.ijo.0803802 (2008).

21. Moon, S. S. Low skeletal muscle mass is associated with insulin resistance, diabetes, and metabolic syndrome in the Korean population: the Korea National Health and Nutrition Examination Survey (KNHANES) 2009-2010. Endocr J 61, 61-70 (2014).

22. Seabolt, L. A., Welch, E. B. \& Silver, H. J. Imaging methods for analyzing body composition in human obesity and cardiometabolic disease. Ann N Y Acad Sci 1353, 41-59, https://doi.org/10.1111/nyas.12842 (2015).

23. Liberati, A. et al. The PRISMA statement for reporting systematic reviews and meta-analyses of studies that evaluate healthcare interventions: explanation and elaboration. BMJ (Clinical research ed.) 339, b2700, https://doi.org/10.1136/bmj.b2700 (2009).

24. von Elm, E. et al. Strengthening the Reporting of Observational Studies in Epidemiology (STROBE) statement: guidelines for reporting observational studies. BMJ (Clinical research ed.) 335, 806-808, https://doi.org/10.1136/bmi.39335.541782.AD (2007).

25. Deeks, J. J. et al. Evaluating non-randomised intervention studies. Health Technol Assess 7(iii-x), 1-173 (2003).

26. Herzog, R. et al. Are healthcare workers' intentions to vaccinate related to their knowledge, beliefs and attitudes? A systematic review. BMC Public Health 13, 154, https://doi.org/10.1186/1471-2458-13-154 (2013).

27. Raska, I. Jr., Raskova, M., Zikan, V. \& Skrha, J. Body composition is associated with bone and glucose metabolism in postmenopausal women with type 2 diabetes mellitus. Physiological research 66, 99-111 (2017).

28. Stoney, R. M. et al. Do postmenopausal women with NIDDM have a reduced capacity to deposit and conserve lower-body fat? Diabetes care 21, 828-830 (1998).

29. Choi, S. I. et al. Relationship between Regional Body Fat Distribution and Diabetes Mellitus: 2008 to 2010 Korean National Health and Nutrition Examination Surveys. Diabetes \& metabolism journal 41, 51-59, https://doi.org/10.4093/dmj.2017.41.1.51 (2017).

30. Neeland, I. J. et al. Dysfunctional adiposity and the risk of prediabetes and type 2 diabetes in obese adults. Jama 308, 1150-1159, https://doi.org/10.1001/2012.jama.11132 (2012).

31. Jung, S. H., Ha, K. H. \& Kim, D. J. Visceral Fat Mass Has Stronger Associations with Diabetes and Prediabetes than Other Anthropometric Obesity Indicators among Korean Adults. Yonsei Med J 57, 674-680, https://doi.org/10.3349/ymj.2016.57.3.674 (2016).

32. Leslie, W. D., Ludwig, S. M. \& Morin, S. Abdominal fat from spine dual-energy x-ray absorptiometry and risk for subsequent diabetes. J Clin Endocrinol Metab 95, 3272-3276, https://doi.org/10.1210/jc.2009-2794 (2010).

33. Nordstrom, A., Hadrevi, J., Olsson, T., Franks, P. W. \& Nordstrom, P. Higher Prevalence of Type 2 Diabetes in Men Than in Women Is Associated With Differences in Visceral Fat Mass. J Clin Endocrinol Metab 101, 3740-3746, https://doi.org/10.1210/jc.2016-1915 (2016).

34. Peterson, M. D., Al Snih, S., Serra-Rexach, J. A. \& Burant, C. Android Adiposity and Lack of Moderate and Vigorous Physical Activity Are Associated With Insulin Resistance and Diabetes in Aging Adults. J Gerontol A Biol Sci Med Sci 70, 1009-1017, https:// doi.org/10.1093/gerona/glv002 (2015).

35. Rothney, M. P. et al. Abdominal visceral fat measurement using dual-energy X-ray: association with cardiometabolic risk factors. Obesity (Silver Spring) 21, 1798-1802, https://doi.org/10.1002/oby.20223 (2013).

36. Akeroyd, J. M. et al. Differences in skeletal and non-skeletal factors in a diverse sample of men with and without type 2 diabetes mellitus. Journal of diabetes and its complications 28,679-683, https://doi.org/10.1016/j.jdiacomp.2014.05.007 (2014).

37. Anbalagan, V. P. et al. The prevalence of presarcopenia in Asian Indian individuals with and without type 2 diabetes. Diabetes Technol Ther 15, 768-775, https://doi.org/10.1089/dia.2013.0068 (2013).

38. Davidson, L. E. et al. Skeletal muscle and organ masses differ in overweight adults with type 2 diabetes. J Appl Physiol (1985) 117, 377-382, https://doi.org/10.1152/japplphysiol.01095.2013 (2014).

39. Guerrero, N. et al. Premature loss of muscle mass and function in type 2 diabetes. Diabetes Res Clin Pract 117, 32-38, https://doi. org/10.1016/j.diabres.2016.04.011 (2016).

40. Kim, K. S. et al. Type 2 diabetes is associated with low muscle mass in older adults. Geriatr Gerontol Int 14(1), 115-121, https://doi. org/10.1111/ggi.12189 (2014).

41. Kim, T. N. et al. Prevalence and determinant factors of sarcopenia in patients with type 2 diabetes: the Korean Sarcopenic Obesity Study (KSOS). Diabetes care 33, 1497-1499, https://doi.org/10.2337/dc09-2310 (2010). 
42. Park, S. W. et al. Excessive loss of skeletal muscle mass in older adults with type 2 diabetes. Diabetes care 32, 1993-1997, https://doi. org/10.2337/dc09-0264 (2009).

43. Renoud, A., Ecochard, R., Marchand, F., Chapurlat, R. \& Szulc, P. Predictive parameters of accelerated muscle loss in men-MINOS study. Am J Med 127, 554-561, https://doi.org/10.1016/j.amjmed.2014.02.004 (2014).

44. Yoon, J. W. et al. Hyperglycemia Is Associated with Impaired Muscle Quality in Older Men with Diabetes: The Korean Longitudinal Study on Health and Aging. Diabetes Metab J 40, 140-146, https://doi.org/10.4093/dmj.2016.40.2.140 (2016).

45. Larsen, B. A. et al. Association of Muscle Mass, Area, and Strength With Incident Diabetes in Older Adults: The Health ABC Study. J Clin Endocrinol Metab 101, 1847-1855, https://doi.org/10.1210/jc.2015-3643 (2016).

46. Li, J. J. et al. Muscle grip strength predicts incident type 2 diabetes: Population-based cohort study. Metabolism 65, 883-892, https:// doi.org/10.1016/j.metabol.2016.03.011 (2016).

47. Saito, T. et al. Association of subcutaneous and visceral fat mass with serum concentrations of adipokines in subjects with type 2 diabetes mellitus. Endocr J 59, 39-45 (2012).

48. Kadowaki, T. et al. Adiponectin and adiponectin receptors in insulin resistance, diabetes, and the metabolic syndrome. JClin Invest 116, 1784-1792, https://doi.org/10.1172/jci29126 (2006).

49. Sheng, T. \& Yang, K. Adiponectin and its association with insulin resistance and type 2 diabetes. J Genet Genomics 35, 321-326, https://doi.org/10.1016/s1673-8527(08)60047-8 (2008).

50. Boden, G. Free fatty acids, insulin resistance, and type 2 diabetes mellitus. Proceedings of the Association of American Physicians 111, 241-248 (1999).

51. Coppack, S. W. Pro-inflammatory cytokines and adipose tissue. Proc Nutr Soc 60, 349-356 (2001).

52. Neeland, I. J. et al. Associations of visceral and abdominal subcutaneous adipose tissue with markers of cardiac and metabolic risk in obese adults. Obesity (Silver Spring) 21, E439-447, https://doi.org/10.1002/oby.20135 (2013).

53. Scheck, S. H. et al. Effects of NIDDM on the glucose transport system in human skeletal muscle. Diabetes research (Edinburgh, Scotland) 16, 111-119 (1991).

54. Taaffe, D. R., Harris, T. B., Ferrucci, L., Rowe, J. \& Seeman, T. E. Cross-sectional and prospective relationships of interleukin-6 and C-reactive protein with physical performance in elderly persons: MacArthur studies of successful aging. The journals of gerontology. Series A, Biological sciences and medical sciences 55, M709-715 (2000).

55. Visser, M. et al. Relationship of interleukin-6 and tumor necrosis factor-alpha with muscle mass and muscle strength in elderly men and women: the Health ABC Study. The journals of gerontology. Series A, Biological sciences and medical sciences 57, M326-332 (2002).

56. Fano, G. et al. Age and sex influence on oxidative damage and functional status in human skeletal muscle. Journal of muscle research and cell motility 22, 345-351 (2001).

57. Barazzoni, R. Skeletal muscle mitochondrial protein metabolism and function in ageing and type 2 diabetes. Current opinion in clinical nutrition and metabolic care 7, 97-102 (2004).

58. Deurenberg, P., Deurenberg-Yap, M. \& Guricci, S. Asians are different from Caucasians and from each other in their body mass index/body fat per cent relationship. Obesity reviews: an official journal of the International Association for the Study of Obesity 3 , $141-146$ (2002).

59. Wang, D. et al. Ethnic differences in body composition and obesity related risk factors: study in Chinese and white males living in China. PloS one 6, e19835, https://doi.org/10.1371/journal.pone.0019835 (2011).

60. Pouliot, M. C. et al. Visceral obesity in men. Associations with glucose tolerance, plasma insulin, and lipoprotein levels. Diabetes 41, 826-834 (1992)

61. Banerji, M. A. et al. Relationship of visceral adipose tissue and glucose disposal is independent of sex in black NIDDM subjects. Am J Physiol 273, E425-432 (1997).

62. Wander, P. L. et al. Change in visceral adiposity independently predicts a greater risk of developing type 2 diabetes over 10 years in Japanese Americans. Diabetes care 36, 289-293, https://doi.org/10.2337/dc12-0198 (2013).

63. Kwon, H., Kim, D. \& Kim, J. S. Body Fat Distribution and the Risk of Incident Metabolic Syndrome: A Longitudinal Cohort Study. Sci Rep 7, 10955, https://doi.org/10.1038/s41598-017-09723-y (2017).

64. Elffers, T. W. et al. Body fat distribution, in particular visceral fat, is associated with cardiometabolic risk factors in obese women. PloS one 12, e0185403, https://doi.org/10.1371/journal.pone.0185403 (2017).

\section{Author Contributions}

P.G.: Systematically searched databases to assess the titles, abstracts and full articles; analysis and interpretation of meta-data; drafting of manuscript. C.L.: Systematically searched databases to assess the titles, abstracts and full articles; review of manuscript. A.G.: Analysis and interpretation of data; review of manuscript. P.S.: Review of manuscript. S.T.: Review of manuscript. Y.T.: Review of manuscript. N.K.: Review of manuscript. R.M.: Review of manuscript. E.F.: Review of manuscript. E.L.: Interpretation of data; revision of article; final approval of version to be published.

\section{Additional Information}

Competing Interests: The authors declare no competing interests.

Publisher's note: Springer Nature remains neutral with regard to jurisdictional claims in published maps and institutional affiliations.

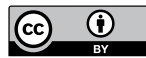

Open Access This article is licensed under a Creative Commons Attribution 4.0 International License, which permits use, sharing, adaptation, distribution and reproduction in any medium or format, as long as you give appropriate credit to the original author(s) and the source, provide a link to the Creative Commons license, and indicate if changes were made. The images or other third party material in this article are included in the article's Creative Commons license, unless indicated otherwise in a credit line to the material. If material is not included in the article's Creative Commons license and your intended use is not permitted by statutory regulation or exceeds the permitted use, you will need to obtain permission directly from the copyright holder. To view a copy of this license, visit http://creativecommons.org/licenses/by/4.0/.

(C) The Author(s) 2019 